International Journal of Pure and Applied Mathematics

Volume 108 No. 4 2016, 887-894

ISSN: 1311-8080 (printed version); ISSN: 1314-3395 (on-line version)

url: http://www.ijpam.eu

doi: $10.12732 /$ ijpam.v108i4.14

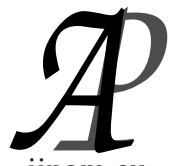

ijpam.eu

\title{
SOME OPERATOR INEQUALITIES FOR $\tau$-MEASURABLE OPERATORS
}

\author{
G.E. Berikhanova ${ }^{1 \S}$, O.M. Zholymbaev ${ }^{2}$, A.A. Anyarov ${ }^{3}$ \\ ${ }^{1,2,3}$ Shakarim State University Of Semey \\ Glinka st. 20A, Semey, KAZAKHSTAN
}

\begin{abstract}
In this present paper we proved some subadditivity submajorisation inequalities for operator concave and operator convex function of $\tau$-measurable operators. Our results extended some results of Hansen [6] and Bourin [1].
\end{abstract}

AMS Subject Classification: 46L10, 46L50, 47D25

Key Words: von Neumann algebra, $\tau$-measurable operator, operator inequality

\section{Preliminaries and Introduction}

In this work considered some operator inequalities for $\tau$ - measurable operators. Our results generalized some operator inequalities which was proved for matrices and bounded operators on Hilbert space. Before showing main results, we need some notions and notations from the theory of $\tau$-measurable operators and non-commutative symmetric spaces.

Let $\mathcal{M}$ be a finite von Neumann algebra acting on a Hilbert space $\mathcal{H}$ with a normalized normal finite faithful trace $\tau$. Let $L_{0}(\mathcal{M})$ denote the topological $*$ algebra of measurable operators with respect to $(\mathcal{M}, \tau)$. The topology of $L_{0}(\mathcal{M})$ is determined by the convergence in measure (see [10]). The trace $\tau$ can be

Received: June 4, 2016

Published: August 16, 2016

${ }^{\S}$ Correspondence author
(C) 2016 Academic Publications, Ltd.

url: www.acadpubl.eu 
extended to the positive cone $L_{0}^{+}(\mathcal{M})$ of $L_{0}(\mathcal{M})$ :

$$
\tau(x)=\int_{0}^{\infty} \lambda d \tau\left(e_{\lambda}(x)\right),
$$

where $x=\int_{0}^{\infty} \lambda d e_{\lambda}(x)$ is the spectral decomposition of $x$.

For $x \in L_{0}(\mathcal{M})$ we define

$$
\lambda_{s}(x)=\tau\left(e_{s}^{\perp}(|x|)\right) \quad(s>0)
$$

and

$$
\mu_{t}(x)=\inf \left\{s>0: \lambda_{s}(x) \leq t\right\} t>0,
$$

where $e_{s}^{\perp}(|x|)=e_{(s, \infty)}(|x|)$ is the spectral projection of $|x|$ associated with the interval $(s, \infty)$. The function $s \mapsto \lambda_{s}(x)$ is called the distribution function of $x$ and $\mu_{t}(x)$ is the generalized s-number of $x$. We will denote simply by $\lambda(x)$ and $\mu(x)$ the functions $s \mapsto \lambda_{s}(x)$ and $t \mapsto \mu_{t}(x)$, respectively. It is easy to check that both are decreasing and continuous from the right on $(0, \infty)$. For further information we refer the reader to [4]. For more details on generalized singular value function of measurable operators we refer to [4].

Given $0<p \leq \infty$ we denote by $L_{p}(\mathcal{M})$ the usual noncommutative $L_{p}$-spaces associated wit $(\mathcal{M}, \tau)$. Recall that $L_{\infty}(\mathcal{M})=\mathcal{M}$, equipped with the operator norm (see [10]). The norm of $L_{p}(\mathcal{M})$ will be denoted by $\|\cdot\|_{p}$. We now recall the definition of a symmetric operator space $E(\mathcal{M})$ buildup with respect to a noncommutative measure space $(\mathcal{M}, \tau)$ and a symmetric Banach function space. Let $(\Omega, \Sigma, \mu)$ be a complete $\sigma$-finite measure space and $L_{0}(\Omega)$ be the space of all classes of $\mu$-measurable real-valued functions defined on $\Omega$. For $f \in L_{0}(0, \infty)$ we denote by $f^{*}$ the decreasing rearrangement of the function $|f|$. Recall that a Banach space $E,\|\cdot\|_{E}$ is called a rearrangement invariant Banach function space if $\{0\} \neq E \subseteq L_{0}(0, \infty)$ and $f \in E, g \in L_{0}(0, \infty), g^{*} \leq f^{*}$ imply that $g \in E$ and $\|g\|_{E} \leq\|f\|_{E}$. Special examples of such Banach function spaces are the spaces $L^{p}(0, \infty), 1 \leq p \leq \infty$ equipped with their usual norm $\|\cdot\|_{p}$. In particular, we recall that any such space satisfies $L^{1} \cap L^{\infty}(0, \infty) \subseteq E \subseteq\left(L_{1}+L_{\infty}\right)(0, \infty)$, with continuous embeddings. The space $E$ is called fully symmetric if, in addition, for $f \in L_{0}(0 ; 1)$ (or $(0, \infty)$ ) and $g \in E$ with $f \preceq g$ implies that $f \in E$ and $\|f\|_{E} \leq\|g\|_{E}$. A rearrangement invariant Banach function space $E$ will be called symmetric, if $f, g \in E$ and $g \preceq f$ imply $\|g\|_{E} \leq\|f\|_{E}$. Here $g \preceq f$ means that the function $g$ is submajorized by $f$, i.e.,

$$
\int_{0}^{t} g^{*}(s) d s \leq \int_{0}^{t} f^{*}(s) d s
$$


for all $t>0$. For more details on symmetric spaces and rearrangements we refer to $[9]$.

Definition 1. A function $f$ is said to be operator convex (see for more details [11]) if $x, y$ be self-adjoint $\tau$-measurable operator and for all real numbers $0 \leq \lambda \leq 1$,

$$
f((1-\lambda) x+\lambda y) \leq(1-\lambda) f(x)+\lambda f(y) .
$$

Several interesting inequalities for operator convex, operator concave and operator monotone functions have recently established in a series of papers like [1], [6], [7] and [11]. Most of theirs results have proved for $\tau$-measurable operators. The following inequality

$$
\left\|f\left(\left|Z^{*} A Z\right|\right)\right\| \leq\left\|f\left(Z^{*} f(|A|) Z\right)\right\|
$$

has proved in [1] for normal $n \times n$ matrices $A$ in symmetric norm, where $f(t)$ is a non-negative concave function on $[0, \infty)$. When $x$ is a positive bounded operator and $a$ is a contractive bounded operator on a Hilbert space $\mathcal{H}$, Hansen proved the inequality

$$
a^{*} f(x) a \leq f\left(a^{*} x a\right)
$$

for a operator monotone function $f(t)$ defined on $[0, \infty)$ (see [6]). In this work we extended these results to the $\tau$-measurable operators.

\section{Main results}

Theorem 2. Let $x$ be a self-adjoint $\tau$-measurable operator and $f$ : $[0, \infty) \rightarrow[0, \infty)$ be a continuous increasing concave function such that $f(0)=0$. Let $z^{*} x z$ be a positive operator for any $z \in \mathcal{M}$. Then

$$
f\left(\left|z^{*} x z\right|\right) \preceq z^{*} f(|x|) z .
$$

Proof. It is clear that

$$
x \leq|x|,
$$

where $a \leq b$ means that $\langle(b-a) \xi, \xi\rangle \geq 0$ for any $\xi \in \mathcal{H}$ (see [11]), and so, by Proposition 4.5 (iii) in [3] it follows that

$$
z^{*} x z \leq z^{*}|x| z .
$$

On the other hand by Lemma 2.5. (ii) and (iii) in [4], we obtain

$$
\mu_{s}\left(\left|z^{*} x z\right|\right)=\mu_{s}\left(z^{*} x z\right) \leq \mu_{s}\left(z^{*}|x| z\right)
$$


Applying Lemma 2.5. (iv) in [4], we get

$$
\mu_{s}\left(f\left(\left|z^{*} x z\right|\right)\right) \leq \mu_{s}\left(f\left(z^{*}|x| z\right)\right)
$$

Hence, by Theorem 3.2.8 in [2], we have that

$$
f\left(\left|z^{*} x z\right|\right) \preceq f\left(z^{*}|x| z\right) \preceq z^{*} f(|x|) z
$$

which is completes of proof.

Theorem 3. Let the conditions of Theorem 2 hold. Then we have

$$
\left(\begin{array}{cc}
f\left(\left|z^{*} x z\right|\right) & 0 \\
0 & f\left(\left|z^{*} x^{*} z\right|\right)
\end{array}\right) \preceq\left(\begin{array}{cc}
z^{*} f(|x|) z & 0 \\
0 & z^{*} f\left(\left|x^{*}\right|\right) z
\end{array}\right) .
$$

Proof. Let consider a hermitian operator

$$
H=\left(\begin{array}{cc}
0 & x^{*} \\
x & 0
\end{array}\right)
$$

Applying Theorem 2. to the $H$ we obtain

$$
\begin{aligned}
& f\left(\left|\left(\begin{array}{cc}
z & 0 \\
0 & z
\end{array}\right)^{*}\left(\begin{array}{cc}
0 & x^{*} \\
x & 0
\end{array}\right)\left(\begin{array}{ll}
z & 0 \\
0 & z
\end{array}\right)\right|\right) \\
& \preceq\left(\begin{array}{cc}
z & 0 \\
0 & z
\end{array}\right)^{*} f\left(\left|\left(\begin{array}{cc}
0 & x^{*} \\
x & 0
\end{array}\right)\right|\right)\left(\begin{array}{cc}
z & 0 \\
0 & z
\end{array}\right)
\end{aligned}
$$

Hence, by an elementary calculation we get

$$
\begin{gathered}
\left(\begin{array}{cc}
f\left(\left|z^{*} x z\right|\right) & 0 \\
0 & f\left(\left|z^{*} x^{*} z\right|\right)
\end{array}\right)=f\left(\left(\begin{array}{cc}
z^{*} x z \mid & 0 \\
0 & \left|z^{*} x^{*} z\right|
\end{array}\right)\right) \\
=f\left(\left|\left(\begin{array}{cc}
0 & z^{*} x^{*} z \\
z^{*} x z & 0
\end{array}\right)\right|\right)=f\left(\left|\left(\begin{array}{cc}
z & 0 \\
0 & z
\end{array}\right){ }^{*}\left(\begin{array}{cc}
0 & x^{*} \\
x & 0
\end{array}\right)\left(\begin{array}{cc}
z & 0 \\
0 & z
\end{array}\right)\right|\right) \\
\preceq\left(\begin{array}{cc}
z & 0 \\
0 & z
\end{array}\right)^{*} f\left(\left|\left(\begin{array}{cc}
0 & x^{*} \\
x & 0
\end{array}\right)\right|\right)\left(\begin{array}{cc}
z & 0 \\
0 & z
\end{array}\right)=\left(\begin{array}{cc}
z^{*} & 0 \\
0 & z^{*}
\end{array}\right) \\
\cdot f\left(\left(\begin{array}{cc}
|x| & 0 \\
0 & \left|x^{*}\right|
\end{array}\right)\right)\left(\begin{array}{cc}
z & 0 \\
0 & z
\end{array}\right)=\left(\begin{array}{cc}
z^{*} & 0 \\
0 & z^{*}
\end{array}\right)\left(\begin{array}{cc}
f(|x|) & 0 \\
0 & f\left(\left|x^{*}\right|\right)
\end{array}\right) \\
\cdot\left(\begin{array}{cc}
z & 0 \\
0 & z
\end{array}\right)=\left(\begin{array}{cc}
z^{*} f(|x|) z & 0 \\
0 & z^{*} f\left(\left|x^{*}\right|\right) z
\end{array}\right)
\end{gathered}
$$

This completes the proof. 
Theorem 4. Let $y$ be a positive $\tau$-measurable operator and $a \in \mathcal{M}$ be a contraction.

(i) If $f$ is a nonnegative operator monotone function on $[0, \infty)$, then

$$
a^{*} f(y) a \leq f\left(a^{*} y a\right)
$$

(ii) If $g$ is a nonnegative operator convex function on $[0, \infty)$ and $g(0) \leq 0$, then

$$
g\left(a^{*} y a\right) \leq a^{*} g(y) a .
$$

Proof. (i) We take the linear operator $U$ on $\mathcal{H} \oplus \mathcal{H}$ given by

$$
U=\left(\begin{array}{cc}
a & c \\
-b & a^{*}
\end{array}\right),
$$

where $b=\left(1-a^{*} a\right)^{\frac{1}{2}}$ and $c=\left(1-a a^{*}\right)^{\frac{1}{2}}$. It is proved in [5] that $U$ is unitary (see [5], solution 177). Let

$$
Y=\left(\begin{array}{ll}
y & 0 \\
0 & 0
\end{array}\right)
$$

and observe that

$$
U^{*} Y U=\left(\begin{array}{cc}
a^{*} y a & a^{*} y c \\
c y a & c y c
\end{array}\right)
$$

Consider now a constant $\varepsilon>0$ and set

$$
X=\left(\begin{array}{cc}
a^{*} y a+\varepsilon & 0 \\
0 & 2 \lambda
\end{array}\right),
$$

where $\lambda$ is a positive constant. Then we easily obtain that

$$
\begin{array}{r}
X-U^{*} Y U=\left(\begin{array}{cc}
\varepsilon & -a^{*} y c \\
-c y a & 2 \lambda-c y c
\end{array}\right) \\
\geq\left(\begin{array}{cc}
\varepsilon & d \\
d^{*} & \lambda
\end{array}\right), \quad \text { for } \lambda \geq c y c
\end{array}
$$

where $d=-a^{*} y c$. Let $\xi, \eta \in \mathcal{H}$, then we have 


$$
\begin{array}{r}
\left\langle\left(\begin{array}{cc}
\varepsilon & d \\
d^{*} & \lambda
\end{array}\right)\left(\begin{array}{c}
\xi \\
\eta
\end{array}\right),\left(\begin{array}{c}
\xi \\
\eta
\end{array}\right)\right\rangle \\
=\varepsilon\|\xi\|^{2}+\langle d \eta, \xi\rangle+\left\langle d^{*} \xi, \eta\right\rangle+\lambda\|\eta\|^{2} \\
\geq \varepsilon\|\xi\|^{2}-2\|d\|\|\xi\|\|\eta\|+\lambda\|\eta\|^{2} \\
\geq 0 \text { for } \lambda \geq \frac{\|d\|^{2}}{\varepsilon} .
\end{array}
$$

for a large reasonably $\lambda$. So we observe that

$$
U^{*} Y U \leq X
$$

Hence we get

$$
U^{*} f(Y) U=f\left(U^{*} Y U\right) \leq f(X)
$$

or

$$
\left(\begin{array}{cc}
a^{*} f(y) a & a^{*} f(y) c \\
c f(y) a & c f(y) c
\end{array}\right) \leq\left(\begin{array}{cc}
f\left(a^{*} y a+\varepsilon\right) & d \\
d^{*} & f(2 \lambda)
\end{array}\right) .
$$

In particular we have $a^{*} f(y) a \leq f\left(a^{*} y a+\varepsilon\right)$. Since operator monotone function is continuous and $\varepsilon$ is arbitrary, letting $\varepsilon \rightarrow 0$ we get the desired inequality.

(ii) Let put linear operators $U$ and $V$ on $\mathcal{H} \oplus \mathcal{H}$ as follows

$$
U=\left(\begin{array}{cc}
a & c \\
-b & a^{*}
\end{array}\right), V=\left(\begin{array}{cc}
a & -c \\
b & a^{*}
\end{array}\right)
$$

where $b=\left(1-a^{*} a\right)^{\frac{1}{2}}$ and $c=\left(1-a a^{*}\right)^{\frac{1}{2}}$. Then it is clearly that $U$ and $V$ are unitary operators. Let

$$
Y=\left(\begin{array}{ll}
y & 0 \\
0 & 0
\end{array}\right)
$$

and note that

$$
U^{*} Y U=\left(\begin{array}{cc}
a^{*} y a & a^{*} y c \\
c y a & c y c
\end{array}\right), V^{*} Y V=\left(\begin{array}{cc}
a^{*} y a & -a^{*} y c \\
-c y a & c y c
\end{array}\right) .
$$

Since $g$ is operator convex, we have that 


$$
\begin{aligned}
& \left(\begin{array}{cc}
g\left(a^{*} y a\right) & 0 \\
0 & g(b x b)
\end{array}\right)=g\left(\begin{array}{cc}
a^{*} y a & 0 \\
0 & c y c
\end{array}\right)=g\left(\frac{1}{2} U^{*} X U+\frac{1}{2} V^{*} X V\right) \\
\leq & \frac{1}{2} g\left(U^{*} X U\right)+\frac{1}{2} g\left(V^{*} X V\right)=\frac{1}{2} U^{*} g(X) U+\frac{1}{2} V^{*} g(X) V \\
= & \frac{1}{2} U^{*} g\left(\begin{array}{cc}
y & 0 \\
0 & 0
\end{array}\right) U+\frac{1}{2} V^{*} g\left(\begin{array}{cc}
y & 0 \\
0 & 0
\end{array}\right) V=\frac{1}{2} U^{*}\left(\begin{array}{cc}
g(y) & 0 \\
0 & g(0)
\end{array}\right) U \\
+ & \frac{1}{2} V^{*}\left(\begin{array}{cc}
g(y) & 0 \\
0 & g(0)
\end{array}\right) V \leq \frac{1}{2} U^{*}\left(\begin{array}{cc}
g(y) & 0 \\
0 & 0
\end{array}\right) U+\frac{1}{2} V^{*}\left(\begin{array}{cc}
g(y) & 0 \\
0 & 0
\end{array}\right) V \\
= & \left(\begin{array}{cc}
a^{*} g(y) a & 0 \\
0 & c g(y) c
\end{array}\right) .
\end{aligned}
$$

In particular, we get $g\left(a^{*} y a\right) \leq a^{*} g(y) a$. This completes the proof.

Specializing Theorem 4 to the functions $f(t)=t^{p}(1 \leq p<\infty)$ and $g(t)=$ $t^{p}(0<p \leq 1)$, respectively, we obtain the following result.

Corollary 5. Let $y$ be a positive $\tau$-measurable operator, $a \in \mathcal{M}$ be a contraction, then

$$
\begin{aligned}
& a^{*} y^{r} a \leq\left(a^{*} y a\right)^{r}, \text { for each } 0<p \leq 1 \\
& \left(a^{*} y a\right)^{r} \leq a^{*} y^{r} a, \text { for } \text { each } 1 \leq p<\infty
\end{aligned}
$$

Above result was proved by Kosaki in [8] when $0<p \leq 1$. In particular, when $a$ is a projection, we have the following Corollary.

Corollary 6. Let $y$ be a positive $\tau$-measurable operator, $e \in \mathcal{M}$ be a projection, then

$$
\begin{aligned}
& e^{*} y^{r} e \leq\left(e^{*} y e\right)^{r}, \text { for each } 0<p \leq 1 \\
& \left(e^{*} y e\right)^{r} \leq e^{*} y^{r} e, \text { for } \text { each } 1 \leq p<\infty
\end{aligned}
$$

\section{References}

[1] J.-C. Bourin, A matrix subadditivity inequality for symmetric norms, Proc. AMS $\mathbf{1 3 8}$ (2010), 495-504. http://dx.doi.org/10.1090/S0002-9939-09-10103-X

[2] Turdebek N. Bekjan, Dostilek Dauitbek, Submajorization inequalities of $\tau$-measurable operators, ICAAM, AIP Proceedings 1611 (2014), 145-149. http://dx.doi.org/10.1063/1.4893820 
[3] P.G. Dodds, T.K. Dodds and B. de Pager, Non-Commutative Banach function spaces, Math. Z. 201 (1989) 583-587. http://dx.doi.org/10.1007/BF01215160

[4] T. Fack and H. Kosaki, Generalized s-numbers of $\tau$-measure operators,Pac. J. Math. 123 (1986), 269-300. http://dx.doi.org/10.2140/pjm.1986.123.269

[5] P.R. Halmos, A hilbert space problem book, Amer. book, (1967). http://dx.doi.org/10.1007/978-1-4615-9976-0

[6] F. Hansen, An operator inequality, Math. Ann., 246 (1980), 249-250. http://dx.doi.org/10.1007/BF01371046

[7] F. Hansen and G. K. Pedersen, Jensens inequality for operators and Löwners theorem, Math. Ann, 258 (1982), 229-241. http://dx.doi.org/10.1007/BF01450679

[8] H. Kosaki, On the Continuity of the Map $\varphi \rightarrow|\varphi|$ from the Predual of a $W^{*}$-Algebra, $J$. Funct. Anal. 59 (1984), 123-131. http://dx.doi.org/10.1016/0022-1236(84)90055-7

[9] J. Lindenstrauss and L. Tzafriri Classical Banach spaces I and II, Springer-Verlag, Berlin (1979). http://dx.doi.org/10.1007/978-3-540-37732-0

[10] G. Pisier and Q. Xu, Noncommutative $L^{\mathrm{p}}$-spaces, Handbook of the geometry of Banach spaces, vol.2 (2003), 1459-1517. http://dx.doi.org/10.1016/S1874-5849(03)80041-4

[11] X. Zhan, Matrix Inequalities, In Lecture Notes in Mathematics 1790, Berlin: Springer, (2002). http://dx.doi.org/10.1007/b83956 